Reprod. Nutr. Dévelop., 1980, 20 (6), 1849-1854.

\title{
Aspects qualitatifs de la digestion intestinale des glucides d'un tourteau de soja par le veau préruminant
}

par J. M. BESLE, P. THIVEND

Laboratoire de la Digestion des Ruminants, I. N. R. A., Theix, Saint-Genès-Champanelle,

63110 Beaumont, France.

Summary. Qualitative aspects of intestinal digestion of soya carbohydrates in the preruminant calf.

Two 15-day old preruminant calves, fitted with reentrant ileocaecal and single colon cannulae, were fed a milk substitute containing 47 p. 100 of toasted soya oil meal, 17 p. 100 of crystallized tallow, 28.5 p. 100 of lactose, 7.0 p. 100 of minerals plus vitamins and 0.5 p. 100 of DL-methionine all on a dry basis. Samples from the ileum, colon and faeces were collected every 2 hrs for 8 hrs after feeding, when the calves 1 and 2 were 4 and 9 week-old, respectively. Total water-soluble carbohydrates were determined. then identified by ion-exchange chromatography. In this preliminary work, we only present results on the healthiest calf.

At the end of the small intestine, sucrose and $\alpha$-galactosides were probably poorly digested. Their concentration (as a percentage of total water-soluble carbohydrates) was the same as in the soya bean. In the large intestine, these carbohydrates were completely utilized and the sucrose was break down faster than the $\alpha$-galactosides. The end product of fermentation was mainly lactic acid (4-8 $\mathrm{g} / \mathrm{l}$ of intestinal contents), and the $\mathrm{pH}$ values of the large intestine contents were low (4.0-4.9), but these fermentations did not cause digestive problems, such as diarrhea, probably because the amount of soya carbohydrates in the food was low, and the digestive processes along the digestive tract were very regular.

\section{Introduction.}

Après élimination des facteurs antinutritionnels, l'utilisation du tourteau de soja dans les aliments d'allaitement pose encore un certain nombre de problèmes. Les travaux de Paruelle et al. (1972) et de Guilloteau et al. $(1977,1979)$ indiquent que l'absence de coagulation dans la caillette et une possible réaction antigénique (Van Adrichem ef Frens, 1965) ne sont pas les seules raisons de l'utilisation médiocre de cet aliment par le veau préruminant. Elle pourrait également provenir de la fraction glucidique hydrosoluble (environ 13 p. 100 de la matière sèche), formée principalement de saccharose et d' $\alpha$-galactosides (notamment raffinose, stachyose, verbascose). Ces glucides ne peuvent être hydrolysés par voie enzymatique endogène dans l'intestin du veau ef leur dégradation microbienne entraîne une production de gaz qui provoquent des flatulence (Cristofaro, Mottu et Wuhrmann, 1974), irritent 
la muqueuse intestinale et modifient le transit (Hellendoorn, 1969). Ces glucides représentent toutefois une source d'énergie non négligeable et leur élimination de la graine de soja ou de ses dérivés proposée par certains auteurs (Colvin ef Ramsey, 1968 ; Hellendoorn, 1969 ; Lee Kim et Ho, 1972) est toujours coûteuse. Aussi, nous avons réalisé une éfude préliminaire afin de préciser leur mode de digestion dans l'intestin du veau préruminant.

\section{Matériels et méthodes.}

Deux veaux préruminants ont été munis à l'âge de 15 jours, d'une canule réentrante au niveau iléocæcal et d'une canule simple dans le côlon proximal. L'aliment d'allaitement du commerce qu'ils recevaient depuis leur naissance a été remplacé en 10 jours par un régime contenant par rapport à la matière sèche, 47 p. 100 de tourteau de soja cuit, 17 p. 100 de suif cristallisé, 28,5 p. 100 de lactose, 0,5 p. 100 de DLméthionine ef 7 p. 100 de minéraux et de vitamines. Le soja a été incorporé à un taux volontairement élevé afin d'apporter des quantités relativement importantes d' $\alpha$-galatosides et saccharose ( 6 p. 100 de la matière sèche au tolal) ; il fournissaif la quasi-totalité des protéines dans l'aliment. Celui-ci était distribué sous forme liquide à raison de $130 \mathrm{~g} / \mathrm{kg}$ de lait reconstitué. Les animaux étaient alimentés au seau, en 2 repas par jour, les quantités augmentant tous les 15 jours en fonction de leur poids métabolique $\left(P^{0,75}\right)$. Les veaux 1 et 2 étaient âgés respectivement de 4 et 9 semaines lorsque les prélèvements ont commencé. Ceux-ci ont été effectués trois jours de suite toutes les $2 \mathrm{~h}$ pendant $8 \mathrm{~h}$ après le repas du matin, d'abord dans le côlon (premier jour) puis sur les fèces et enfin dans l'iléon selon les modalités décrites par Assan (1974).

Les échantillons pour le dosage des glucides ont été stabilisés dans l'azote liquide immédiatement après la collecte, puis lyophilisés. Les conditions d'extraction aqueuse, de purification et de dosage des glucides totaux sont analogues à celles décrites précédemment dans une autre publication (Besle, Lassalas et Thivend, 1980). Nous avons dosé les glucides neutres sur l'extrait purifié et « l'équivalent glucidique total 》 (dénomination préférée à 《 glucides totaux 》) dans les extraits aqueux non purifiés, par rapport à une gamme étalon de glucose. L'erreur sur la valeur réelle ne dépasse pas 10 p. 100 puisque les coefficients de réponse des constituants principaux (saccharose, raffinose et stachyose) sont proches de celui du glucose (respectivement 90,92 ef 93 p. 100). La composition glucidique est déterminée par chromatographie échangeuse d'ions (Besle, 1974). En outre, le saccharose et les $\alpha$-galactosides sont dosés spécifiquement en évaluant le fructose (Besle, 1977) sur une partie de l'effluent de colonne, collecté avec une fractionneuse.

La matière sèche a été déterminée par lyophilisation suivie d'un séchage à l'étuve à $80^{\circ} \mathrm{C}$ pendant $48 \mathrm{~h}$. L'acide lactique total a été dosé sur le produit frais par la méthode de Barker ef Summerson (1941).

\section{Résultats et discussion.}

Etat sanitaire des animaux. - L'aliment a été mal accepté par les animaux, vraisemblablement à cause de sa trop grande richesse en soja. De plus, le mode d'incorporation du suif dans l'aliment (matières grasses cristallisées) n'a pas favorisé une 
bonne homogénéisation du lait reconstitué. En outre l'aliment présentait un taux de furazolidone accidentellement majoré ( 380 ppm au lieu de 50), et une activité antitrypsique trop élevée, celle-ci due sans doute à une cuisson insuffisante du soja. L'appétit des animaux a été faible et leur état sanitaire s'est détérioré au bout de 20 jours d'expérience bien qu'ils n'aient jamais eu de diarrhée. Pour ces différentes raisons, les prélèvements effectués alors que les animaux ingéraient l'un 960 et l'autre $1060 \mathrm{~g} / \mathrm{jour}$ (4 à 14 jours après le début de l'essai) n'ont pas permis de quantifier la digestion. Nous présentons dans cette étude préliminaire, uniquement les résultats concernant la composition glucidique des contenus intestinaux obtenus sur le veau ayant eu le meilleur état sanitaire el une croissance satisfaisante.

Digestion des glucides. - Au niveau de l'iléon (tabl. 1), la concentration en glucides hydrosolubles neutres est importante (14,4 p. 100 de la matière sèche du contenu digestif, $3 \mathrm{~h} 30$ après le repas). Elle augmente ultérieurement ( 32,7 p. 100 au bout de 7 h), vraisemblablement par suite de l'absorption des autres constifuants du régime. Le saccharose, le raffinose et le stachyose constituent les principaux glucides de cette fraction ; leur répartition est sensiblement la même que dans le tourteau de soja. Le lactose est entièrement digéré et ses produits d'hydrolyse sont d'ailleurs presque tolalement absorbés dès leur formation. Sept heures après le repas, il apparaît du glucose, du galactose et un glucide élué du même niveau que le mélibiose qui proviennent vraisemblablement d'un début d'hydrolyse des $\alpha$-galactosides. On observe également de l'arabinose issu sans doute de la dégradation des hémicelluloses. On note aussi la présence d'un glucide, élué dans le chromatogramme au même niveau que le mannose qui pourrait être du verbascose mais que nous n'avons pu identifier.

- Au début du côlon, les glucides neutres hydrosolubles (de 21 à 24 p. 100 de la matière sèche) sont principalement du saccharose, du raffinose et du stachyose. On remarque que la part du saccharose à fortement diminué (18-14 p. 100), celle du raffinose et du stachyose ayant été peu modifiée. Les microorganismes seraient donc plus aptes à dégrader le saccharose que les $\alpha$-galactosides. L'absence de fructose laisse supposer qu'il est très rapidement utilisé par les microorganismes. Enfin, 8 h 30 après le repas, on note un accroissement de la part du glucose, du galactose, du mélibiose (?) et de l'arabinose qui, comme au niveau de l'iléon, proviennent de l'hydrolyse des $\alpha$-galactosides et des hémicelluloses. La forte baisse du pH $(4,0$ à 4,9$)$ et la concentration importante d'acide lactique (4 à $8 \mathrm{~g} / \mathrm{l})$ traduisent une activité fermentaire intense.

- Dans les fèces, il ne reste que 1 à 4 p. 100 de glucides hydrosolubles neutres dont une faible part a pu être déterminée par chromatographie. Le saccharose et les $\alpha$-galactosides du soja sont pratiquement absents, on y trouve surtout (1) du glucose et du galactose (à $7 \mathrm{~h} 30$ et dans des proportions de 1 à 3 soit comme dans le stachyose) qui proviendraient de l'hydrolyse des $\alpha$-galactosides (2) du ribose sans doute produit de dégradation des acides nucléiques des corps microbiens (3) de nombreux pics inconnus.

L'équivalent glucidique total et les glucides neutres suivent une évolution parallèle le long du tube digestif. Les glucides neutres semblent cependant être plus rapidement utilisés car leur concentration relative est plus faible dans les fèces que dans l'iléon. 


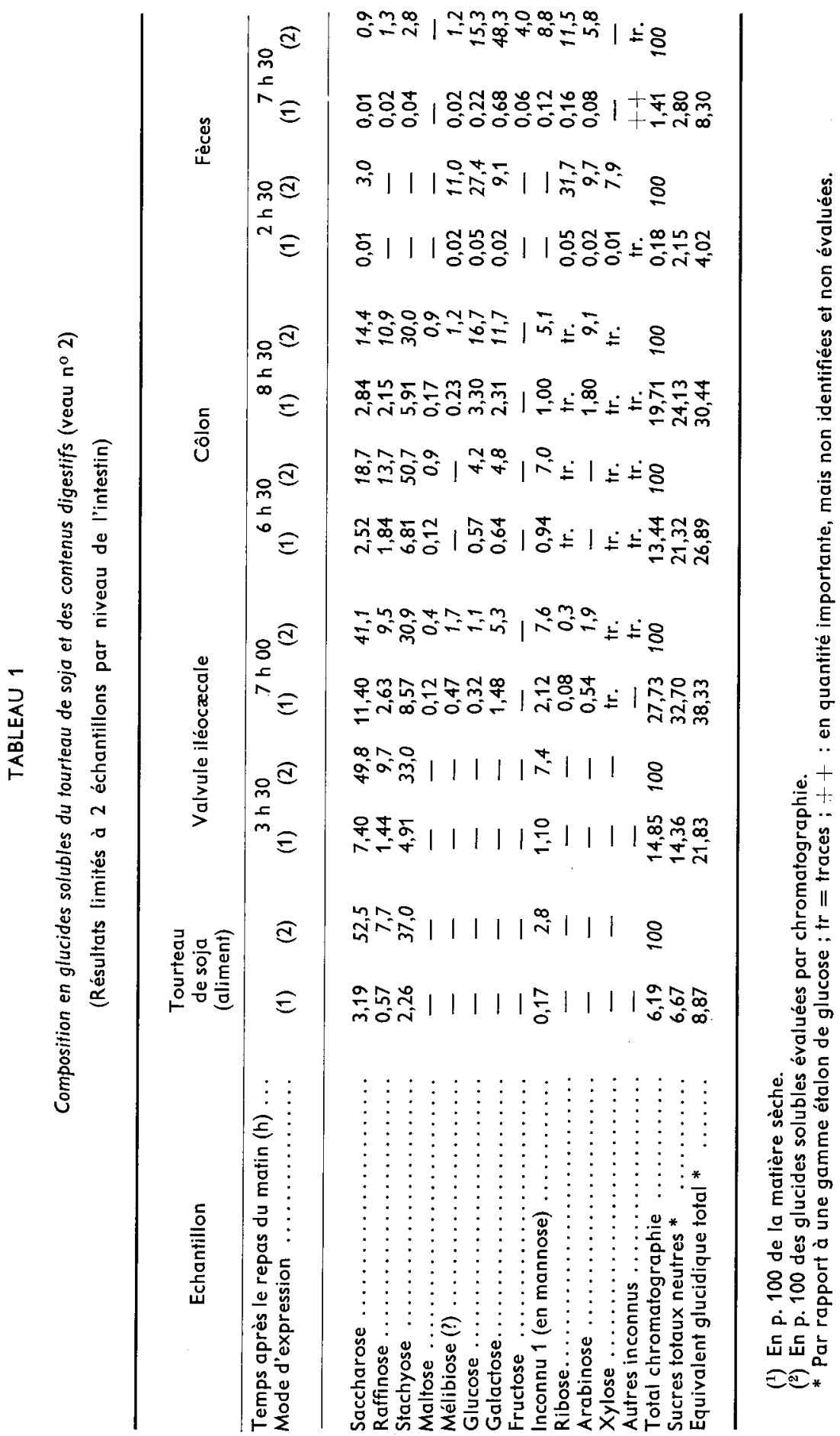


Le veau préruminant est donc capable d'utiliser la totalifé du saccharose et des $\alpha$-galactosides inférieurs du soja (il y a peut-être des $\alpha$-galactosides de poids moléculaire élevé qui résistent). Leur digestion est faible dans l'intestin grêle, elle a lieu principalement dans le gros intestin grâce à la flore microbienne. Le développement des fermentations à ce niveau ne provoque pas la diminution de la teneur en matière sèche des fèces observés avec des régimes contenant des quantités plus importantes de saccharose (Mathieu ef Barré, 1968) ou de certains amidons (Thivend, 1979). Ceci est peut-être dû au fait que les glucides sont ingérés en faibles quantités el qu'ils arrivent régulièrement dans le caecum (Assan, 1974). L'élimination des $\alpha$-galactosides (Guilloteau et al., 1977) et des autres glucides du soja (Porter et Hill, 1963) permet cependant d'améliorer le coefficient d'utilisation digestive apparente de l'azote respectivement de 6 et 13 points. Dans ce dernier cas, il est possible que cette augmentation importante soit due également à un ralentissement du transit dans l'intestin grêle (résultant de l'absence des glucides pariétaux). Toutefois, cette amélioration n'est pas systématique et varie suivant le traitement appliqué pour concentrer les protéines. Il est donc probable que les glucides solubles ne soient pas responsables, même indirectement, de l'utilisation modeste des protéines du soja. En effet, celles-ci, bien que traitées à la chaleur, ont une hydrolyse gastrique moins prononcée que celle du lait ef de plus peuvent entraîner des réactions antigéniques inflammatoires au niveau de la muqueuse de l'intestin grêle (Kilshaw et Sisson, 1979). Ce travail préliminaire qui précise l'importance et le lieu de la digestion des $\alpha$-galactosides devrait permettre d'orienter une étude plus complète pour vérifier les hypothèses avancées.

Reçu en juillet 1978

Accepté en juin 1980.

Remerciements. - A J. P. Brion, J. Lefaivre et A. Vizet qui ont participé à ce travail.

\section{Références}

ASSAN B. E., 1974. Contribution à l'étude de lo digestion intestinale de l'amidon chez le veau préruminant. Th. Fac. Sci. Clermont-Ferrand.

BARKER S. B., SUMMERSON W. H., 1941. The colorimetric determination of lactic acid in biological material. J. biol. Chem., 138, 535-554.

BESLE J. M., 1974. Séparation des oses, des di- et triholosides par chromatographie d'échanges d'ions. Ann. Biol. anim. Bioch. Biophys., 14, 545-573.

BESLE J. M., 1977. Aufomatisation d'une méthode de dosage du fructose, application à l'étude des oligosides du soja. Ann. Biol. anim. Bioch. Biophys., 17, 43-53.

BESLE J. M., LASSALAS B., THIVEND P., 1980. Digestion des glucides de la levure d'alcanes par le veau préruminant. Reprod. Nutr. Dévelop., 20, 1401-1414.

COLVIN B. M., RAMSEY H. A., 1968. Soy flour in milk replacers for young calves. J. Dairy Sci., 51, 898-904.

CRISTOFARO E., MOTTU F., WUHRMANN J. J., 1974. Involvement of the raffinose family of oligosaccharides in flatulence, 313-336, In SIPPLE H. L., Mc NUTT K. W., Sugars in nutrition. Acad. Press, New York, San Francisco, London.

GUILLOTEAU P., TOULLEC R., CULIOLI J., LE DOUARON D., 1977. Utilisation des protéines par le veau préruminant à l'engrais. 5. Utilisation digestive des protéines de poisson, du soja et de la féverole. Ann. Zootech., 26, 15-28.

HELLENDOORN E. W., 1969. Intestinal effects following ingestion of beans. Food Technol., 23, 87-92. 
KILSHAW P. J., SISSONS J. W., 1979. Gastrointestinal allergy to soybean protein in preruminant calves. Antibody production and digestive disturbances in calves fed heated soya bean flour. Res. vet. Sci., 27, 361-365.

LEE Y. H., KIM S. H., HO M. J., 1972. Improvement of nutritive value of soybean bran by fermentation. Korean J. Nutr., 5, 33-42.

MATHIEU C. M., BARRE P. E., 1968. Digestion et utilisation des aliments par le veau préruminant à l'engrais. IV. Remplacement des matières grasses du lait par du saccharose. Ann. Biol. anim. Bioch. Biophys., 8, 501-515.

PARUELLE J. L., TOULLEC R., FRANTZEN J. F., MATHIEU C. M., 1972. Utilisation des protéines par le veau préruminant à l'engrais. 1. Utilisation digestive des protéines de soja et des levures d'alcanes incorporées dans les aliments d'allaitement. Ann. Zoofech., 21, 319-331.

PORTER J. W. G., HILL W. B., 1963. Nitrogen balance trials with calves given synthetic milk diets. Nat. Inst. Res. Dair (Reading). Ann. Rep., 126.

THIVEND P., 1979. La digestion des glucides chez le veau non sevré. Ann. Nutr. Alim., 33, 233-245.

VAN ADRICHEM P. W. M., FRENS A. M., 1965. Soy bean protein as alimentary antigen in fattening calves. Tijdschr. Diergeneesk, 90, 825-830. 